\title{
Imaging Techniques for Evaluation of Drug Distribution in Solid Tumor
}

\author{
Jianfen $\mathrm{Gu}^{1}$, Ningfang Jiang ${ }^{1}$, Huanrong $\mathrm{Lan}^{2}$, Ketao $\mathrm{Jin}^{1}$
}

'Department of Gastrointestinal Surgery, Shaoxing People's Hospital, Shaoxing Hospital of Zhejiang University, Shaoxing, 312000, Zhejiang Province, P.R. China 2Department of Breast and Thyroid Surgery, Shaoxing People's Hospital, Shaoxing Hospital of Zhejiang University, Shaoxing, 312000, Zhejiang Province, P. R. China

\section{ABSTRACT}

Imaging drug distribution inside a tumor is an important tool to support strategies to improve penetration of anticancer drugs and consequently the outcome of chemotherapy. Methods for mapping the distribution of pharmaceutical compounds and their metabolites in situ are of great interest to researchers and to the pharmaceutical industry. There is a particular interest in the distribution of anti-cancer agents within solid tumors. Direct assessment of the distribution of drugs in tumor tissue is technically challenging, and requires the quantification of drug exposure to cells at specific locations relative to blood vessels. The quantification of drugs in plasma, as well as in tissue homogenates, has relied extensively on well-established analytical methods of high-performance liquid chromatography (HPLC) and liquid chromatography-tandem mass spectrometry (LC-MS-MS). In the last 20 years, imaging techniques such as positron emission tomography (PET), magnetic resonance spectroscopy (MRS), mass spectrometry imaging (MSI), fluorescence microscopy (FM), and autoradiography have evolved as powerful tools for the noninvasive study of drug distribution in vivo as well as for studying drug effects at their target sites. In this article, we review the current state of knowledge of the available imaging techniques to measure intratumor drug distribution.

Key words: autoradiography, drug distribution, fluorescence microscopy (FM), magnetic resonance spectroscopy (MRS), mass spectrometry imaging (MSI), positron emission tomography (PET)

\section{INTRODUCTION}

For many drugs the information on plasma pharmacokinetic (PK) is essential to allow rational and effective administration. It is assumed that the drug concentration in plasma is in equilibrium with the drug concentration in tissues, and thus it is a good predictor of the drug present at the site of action. For anticancer agents the relationship between drug concentration in plasma and in neoplastic tissues, that is, primary tumor and metastases, is more complex since tumors are often poorly and irregularly vascularized and are macroscopically and micro-

\author{
Corresponding author: \\ Dr. Ketao Jin \\ Department of Gastrointestinal Surgery \\ Shaoxing People's Hospital \\ Shaoxing Hospital of Zhejiang University. \\ No. 568, Zhongxing North Road, \\ Shaoxing, 312000, Zhejiang Province, \\ P. R. China \\ E-mail: jinketao2001@zju.edu.cn
}

Received: 05.05.2016

Accepted: 08.06.2016

Copyright (c) Celsius Publishing House 
scopically highly heterogeneous. A complete knowledge of drug tissue distribution is essential in new drug development and it is vital to permit interpretation of pharmacological effects. Most PK studies on anticancer drugs have been performed by determining the concentration of the drug and its metabolites in plasma as a function of time after administration in order to define the volume of distribution, half-life, and clearance. These parameters are important for determining the PK features of a drug, and they can be essential in guiding the choice of the most appropriate dosage schedule and route of administration. However, drug plasma concentrations are not necessarily predictive of concentrations achieved in the tumor. In the clinical literature, few papers have examined the correlation between plasma levels of a drug and its concentrations in the tumor. Practical and ethical reasons explain why such important studies have only seldom been performed. The lack of noninvasive techniques easily applicable to determine intratumor levels of the drug is an obstacle in these studies. In some cases, tumor biopsies may be available, but they are generally limited to a single time interval after treatment and do not allow procurement of kinetic data. Some studies have been performed in the neo-adjuvant chemotherapy setting in which patients are treated with several courses before surgery, and tumor tissues, excised during surgery or endoscopic biopsy, can be used to determine drug distribution. However, in most cases the administration of anticancer agents a few hours before tumor excision is not feasible because of potential postsurgical complications, which explains why most available data on tumor drug distribution have been obtained only in preclinical models.

The quantification of drugs in plasma, as well as in tissue homogenates, has relied extensively on wellestablished analytical methods of high-performance liquid chromatography (HPLC) and liquid chromatography-tandem mass spectrometry (LC-MS-MS). In the last 20 years, imaging techniques such as positron emission tomography (PET), magnetic resonance spectroscopy (MRS), mass spectrometry imaging (MSI), fluorescence microscopy (FM), and autoradiography have evolved as powerful tools for the noninvasive study of drug distribution in vivo. Although these techniques were initially introduced to clinical medicine for diagnostic purposes and for the study of tissue metabolism and blood flow (1), they also opened a unique opportunity for PK research by providing a means for noninvasive measurement of drug distribution from the plasma compartment to anatomically defined regions and for the visualization of the entire pattern of drug distribution in given organs (2).
In this article, we review the current state of knowledge of the available imaging techniques to measure intratumor drug distribution.

\section{POSITRON EMISSION TOMOGRAPHY (PET)}

In clinical practice, PET is an important nuclear imaging technique for diagnosis, staging, and response monitoring of cancer (3). The technique is based on the detection of photons released by annihilation of positrons emitted by radiopharmaceuticals. The emitted positrons pass through tissue and are ultimately annihilated when combined with an electron, resulting in two $511 \mathrm{keV}$ photons emitted in opposite directions. Detectors are arranged in a ring around the tissue of interest, and only triggering events that arrive near-simultaneously at diametrically opposite detectors are recorded. The resulting PET images might yield 3-dimensional information on tissue distribution of the positron emitting molecules with a spatial resolution of 1 to $5 \mathrm{~mm}$ and a maximum temporal resolution of $\sim 30$ seconds. The most commonly employed PET radionuclides are oxygen-15 $\left({ }^{15} \mathrm{O}\right)$, nitrogen-13 $\left({ }^{13} \mathrm{~N}\right)$, carbon-11 $\left({ }^{11} \mathrm{C}\right)$, and fluorine-18 $\left({ }^{18} \mathrm{~F}\right)$. Owing to its comparably long half-life, ${ }^{18} \mathrm{~F}$ is the most attractive PET radioisotope, since it allows for imaging durations of up to 10 hours. A considerable drawback, however, is that relatively few drug molecules contain fluorine in their native structure; consequently, despite the rather short $\mathrm{t}_{1 / 2}$ of ${ }^{11} \mathrm{C}$ (20.4 minutes), the majority of PET/PK experiments have relied on ${ }^{11} \mathrm{C}$-labeled tracer molecules.

PK studies using PET might aid in determining intratumoral drug exposure (4). PET detects radiation emitted by radioisotopes in tissue and was applied to study the tumor distribution of some anticancer drugs such as ${ }^{18} \mathrm{~F}$-fluorouracil, ${ }^{18} \mathrm{~F}$-paclitaxel, ${ }^{11} \mathrm{C}$-carmustine, ${ }^{18} \mathrm{~F}$-tamoxifen, and ${ }^{11} \mathrm{C}$-temozolomide (5). It has been shown that PET analysis of radiolabeled anticancer drugs can reveal important information about the distribution of these drugs in patients. For ${ }^{11} \mathrm{C}$-temozolomide, a study showed its good distribution in brain tumor, consistent with its antitumor activity against glioblastoma (6). ${ }^{18} \mathrm{~F}$-fluorouracil is the most common anticancer drug studied with PET. This is because of the ease of ${ }^{18} \mathrm{~F}$-fluorouracil synthesis and the favorable half-life of fluorine (7). ${ }^{18} \mathrm{~F}$-fluorouracil PET studies can give important information about ${ }^{18} \mathrm{~F}$-fluorouracil biodistribution in tumor and normal tissue (8). A study with ${ }^{18} \mathrm{~F}$-paclitaxel (9) examined the effect of P-gp blockers on paclitaxel biodistribution, while the bio-distribution, bioclearance, and in vivo transformation of ${ }^{13} \mathrm{~N}$-cisplatin 
have also been studied with PET (10). Tumor uptake of ${ }^{18} \mathrm{~F}$-tamoxifen has been studied by PET. The ${ }^{18} \mathrm{~F}$-tamoxifen uptake in tumors with good responses was significantly higher than in those with poor responses (11). Tumor uptake of ${ }^{11} \mathrm{C}-1,3$-bis-(2chloroethyl)-1-nitrosourea (BCNU) was compared between i.v. and intra-arterial administration by means of PET measurements. It appeared that intra-arterial administration of ${ }^{11} \mathrm{C} \mathrm{BCNU}$ resulted in 50 times higher intratumoral concentrations (12). Fourteen patients scheduled for fluorouracil chemotherapy received a PET scan with ${ }^{18} \mathrm{~F}$-fluorouracil as a tracer prior to the initiation of chemotherapy. Patients with a high uptake of the radiolabeled drug were more likely to achieve disease stabilization and a longer survival time (13).

PET has also proven to be a valuable noninvasive means for characterizing established and novel anticancer agents (8). Using PET imaging, it was demonstrated that radioactivity uptake was correlated with response to chemotherapy (13). Using PET imaging, the postulated mechanisms of a newer antineoplastic agent, temozolomide, were recently confirmed in vivo in glioma patients (14).

PET using radiolabeled drugs is a promising method to further understand the clinical behavior of anticancer agents. In addition, it may contribute to better guided treatment planning in individual patients with cancer. This technique has several limitations: not every molecule can be labeled and consequently visualized, and the drug and its metabolites cannot be distinguished from one another (4). Furthermore, PET studies are costly because of the need of on-site access to a cyclotron, involvement of radiochemistry, and the need for a PET camera (5).

\section{MAGNETIC RESONANCE SPECTROSCOPY (MRS)}

Magnetic resonance imaging (MRI) uses radiofrequency pulses and magnetic fields to obtain signals from changes in nuclear magnetic moments. A technique based on the same principle as MRI, but providing a greater degree of molecular characterization is MRS, in which spectroscopic profiles of the chemical constituents within a sample are obtained (15). MRS measurements can be performed serially, thus giving the possibility of PK analysis with a temporal resolution in the order of minutes. Of importance, MRS is capable of resolving different chemical species including metabolites owing to different chemical shifts of the resonance signals. This poses a considerable advantage over nuclear imaging methods such as PET, which record nuclear decay events irrespective of the chemical surrounding of the decaying atom and therefore lump together all compounds labeled with the same radioactive atom. A main limitation of all nuclear MR-based methods is their inherent low sensitivity, which restricts the in vivo applicability mainly to molecules that are present in large concentrations in the human body. MRI has proven to be particularly feasible for fluorinated drugs. Several studies have been published describing brain PK of fluorinated psychiatric medications (16), tumor uptake of anticancer chemotherapeutics, or biodistribution and target tissue PK of fluorinated antibiotics $(17,18)$.

\section{MASS SPECTROMETRY IMAGING (MSI)}

MSI is an emerging technique that enables visualization of every molecule in a two-dimensional space directly in biological tissues (19). MSI recognizes the compound of interest on the basis of its molecular mass and fragmentation pattern, without the need to label the analyte, enabling the detection of parent compound and metabolites simultaneously. MSI links molecular information and the spatial distribution of analytes within a sample. In contrast to most histochemical techniques, MSI can differentiate molecular modifications and does not require labeling of targeted compounds. It is possible to visualize the distribution of biomarkers related to tumor heterogeneity, histotype, or therapeutic response (20). The maximum spatial resolution of this technique is between 20 and $100 \mu \mathrm{m}$, depending on the ion source, the chosen matrix, the diameter of the focus laser spot, and even sample characteristics. MSI data can be easily combined with histochemical data because the same samples analyzed by MSI can undergo histological staining. This new technique, providing spatial molecular information in tumor microenvironments, is promising because it permits the correlation of drug concentrations with the histological and molecular features of the tumor tissue. MSI has some advantages in comparison to other imaging techniques, that is, whole body autoradiography, PET or microscopy imaging. It is a label-free technique with better specificity and provides the possibility to combine histological data with MS ones and to visualize simultaneously the distribution of biomarkers in relation to tumor heterogeneity.

MSI is used in a broad range of applications. The vast majority of studies are focusing on tissue originating from animal models or on human clinical tissue originating from surgical interventions. The targeted 
diseases include Parkinson's (21) and Alzheimer's disease (22) as well as Duchenne muscular dystrophy (23). Numerous studies of clinical tissue focused on cancer, for example prostate cancer (24), breast cancer (25), gastric cancer (26), adenocarcinoma (27) and ovarian cancer (28).

Matrix-assisted laser desorption/ionization mass spectrometry imaging (MALDI-MSI) is a promising technology to determine drug distribution and penetration in solid tumors (29). However, thus far only a few papers have addressed this issue (30). The first reported examination using imaging MALDI-MSI of the distribution of a pharmaceutical compound in animal tissue, was carried out by Caprioli's group in 2003, where the distribution of anti-tumor drugs in mouse tumor tissue as well as in rat brain were described (31). Subsequent studies have been carried out to map a range of compounds in a number of different biological tissues $(22,32)$. Caprioli's group was also able to generate images of the antitumor drug imatinib in a mouse brain glioma using ultra high resolution matrix-assisted laser desorption ionization-Fourier transform ion cyclotron resonance (MALDI-FTICR) (33). Atkinson et al. reported the successful detection of the alkylaminoanthraquinone $A Q 4 N$ (banoxatrone) in tumor xenografts by MALDI-MSI (34). The distribution of the multiple-receptor tyrosine kinase inhibitor AEE788 in rodent prostate cancer models was analyzed by MALDI-MSI (35). The tyrosine kinase inhibitors erlotinib and gefitinib have been analyzed, after depositing single droplets of drugs on tissue slices of planocellular lung carcinoma, adeno-carcinoma and large cell lung carcinoma (36-38). Yasunaga et al. (39) showed that paclitaxel-incorporating micelles deliver more paclitaxel to the tumor and less paclitaxel to normal tissue, as compared with treatment with paclitaxel alone. A simple and reproducible protocol for MALDI-MSI was described to visualize the different distribution of paclitaxel in tumor and normal tissues, related to the dosage schedules and pathological features of the tumors (40).

Yasunaga et al. (39) describing the precise distribution of a drug delivery system drug by MALDI-MSI. They successfully visualized and quantified the distribution of a non-radiolabeled and nonchemically modified drug in various frozen tissue slices microscopically. Their results indicated that the MALDI-IMS technique can be applied to clinical biopsy specimens or surgically resected tissues after neo-adjuvant chemotherapy. In addition, the data obtained by MALDI-IMS can be utilized to facilitate drug design. However this approach has some limitations: it is applicable only to molecules that are ionizable by the MSI process; the interpretation of quantitative data is often complex, especially because of the ion suppression effect and chemical noise from the matrix ions covering the drug ion signal; the sensitivity is quite limited as compared with autoradiography (41).

\section{FLUORESCENCE MICROSCOPY (FM)}

FM has probably given the best information about tissue distribution, thanks to its high sensitivity and spatial resolution (200-300 nm). However, it is based on intrinsic fluorescent properties of the drug under analysis and therefore its application is limited to autofluorescent molecules such as topoisomerase inhibitors (42). In preclinical study, the distribution of doxorubicin, mitoxantrone, and topotecan was analyzed by FM at 10 min after administration in tumor-grafted mice. The authors concluded that the compounds are limited to perivascular regions ( 25-75 $\mu \mathrm{m})(43)$. In clinical study, doxorubicin gradients in the tumors from clinical samples were detected by computerized FM on cryosections (44).

\section{AUTORADIOGRAPHY}

The technique of autoradiography is usually divided into whole-body autoradiography (WBA) and microautoradiography. WBA quantitatively analyzes the distribution of a radioactive substance in the different organs of an entire animal carcass that is sliced into thin cryosections. It has several limitations, such as cost and lack of specificity, because this technique cannot distinguish the drug from its metabolites. Moreover, thickness of sections used for WBA causes superimposition of several cellular and tissue structures, leading to resolution pitfalls and misinterpretation of data (45). In preclinical studies, WBA was applied for studying drug distribution of many chemotherapeutics such as $3 \mathrm{H}$-camptothecin, nimustine, methotrexate, and 5 -fluorouracil in brain and tumor tissues of animal models (41). Due to its limited spatial resolution (200 $\mu \mathrm{m}), \mathrm{WBA}$ is not particularly suitable for investigating intratumor drug distribution. By contrast, microautoradiography is a highresolution tool that is used to obtain spatial localization information of radiolabel drugs at the tissue and cellular levels. The same microscope slides used for microautoradiography can be processed using conventional histological staining protocols (41). Microautoradiography was applied to visualize the tumor distribution of $3 \mathrm{H}$-taxanes (paclitaxel and docetaxel), showing that drug accumula- 
tion is limited to the first $100 \mu \mathrm{m}$ of tissue away from vessels in human xenograft tumor (46).

Autoradiography is the standard methodology for imaging pharmaceutical distribution. This technique provides high sensitivity and good image resolution, but does not permit the distinguishing of drug and metabolites. The cost and timescale involved in generating images is also an issue, i.e. a radiolabeled analog of the pharmaceutical under study needs to be synthesized.

\section{CONCLUSION}

Imaging techniques to investigate drug distribution include MRS, PET, MD, autoradiography, FM, and MSI. MRS, PET, and MD can be applied to monitor drug distribution in organs and tissues in the living body in real time. These techniques generally do not efficiently distinguish differential distribution of drugs inside tumor tissue, thus neglecting its heterogeneity and irregular perfusion because of insufficient spatial resolution or technical shortcomings. MRS and PET are noninvasive but do not produce quantitative data, whereas MD provides information about drug concentration in tissue in a semi-invasive way. Autoradiography, FM, and MSI achieve a sufficient spatial resolution to analyze the distribution of drugs inside specific tissues of interest in relation to the pathophysiological structure evaluated by traditional histological techniques. All these techniques have key strengths and limitations, and should be applied in a complementary fashion depending on need.

\section{Authors' contributions}

JFG and KTJ designed this work, analysed the data and wrote this manuscript. NFJ and HRL were involved in designing this work and in drafting the manuscript. All authors read and approved the final manuscript.

\section{Acknowledgments}

This work was supported by National Natural Science Foundation of China (Grant No. 81374014) and Zhejiang Provincial Medical and Healthy Science and Technology Projects (Grant No. 2013KYA228).

\section{Conflict of interest}

The authors declare that they have no conflict of interest.

\section{REFERENCES}

1. Phelps ME. PET: the merging of biology and imaging into molecular imaging. J Nucl Med. 2000 Apr;41(4):661-81.
2. Brunner M, Langer O, Dobrozemsky G, Muller U, Zeitlinger M, Mitterhauser M, et al. (18F)Ciprofloxacin, a new positron emission tomography tracer for noninvasive assessment of the tissue distribution and pharmacokinetics of ciprofloxacin in humans. Antimicrob Agents Chemother. 2004 Oct;48(10):3850-7.

3. Gambhir SS. Molecular imaging of cancer with positron emission tomography. Nat Rev Cancer. 2002 Sep;2(9):683-93.

4. Brunner M, Langer 0 . Microdialysis versus other techniques for the clinical assessment of in vivo tissue drug distribution. AAPS J. 2006 Apr 14;8(2):E263-71.

5. Boss DS, Olmos RV, Sinaasappel M, Beijnen JH, Schellens JH. Application of PET/CT in the development of novel anticancer drugs. Oncologist. 2008 Jan;13(1):25-38.

6. Rosso L, Brock CS, Gallo JM, Saleem A, Price PM, Turkheimer FE et al. A new model for prediction of drug distribution in tumor and normal tissues: pharmacokinetics of temozolomide in glioma patients. Cancer Res. 2009 Jan 1;69(1):120-7.

7. Harte RJ, Matthews JC, O'Reilly SM, Price PM. Sources of error in tissue and tumor measurements of 5-[18F]fluorouracil. J Nucl Med. 1998 Aug;39(8):1370-6.

8. Gupta N, Price PM, Aboagye EO. PET for in vivo pharmacokinetic and pharmacodynamic measurements. Eur J Cancer. 2002 Nov;38(16): 2094-107.

9. Kurdziel KA, Kiesewetter DO, Carson RE, Eckelman WC and Herscovitch P. Biodistribution, radiation dose estimates, and in vivo Pgp modulation studies of $18 \mathrm{~F}$-paclitaxel in nonhuman primates. J Nucl Med 2003; 44: 1330-1339.

10. Ginos JZ, Cooper AJ, Dhawan V, Lai JC, Strother SC, Alcock N et al. [13N]cisplatin PET to assess pharmacokinetics of intra-arterial versus intravenous chemotherapy for malignant brain tumors. J Nucl Med. 1987 Dec;28(12):1844-52.

11. Inoue T, Kim EE, Wallace S, Yang DJ, Wong FC, Bassa P, et al. Positron emission tomography using [18F]fluorotamoxifen to evaluate therapeutic responses in patients with breast cancer: preliminary study. Cancer Biother Radiopharm. 1996 Aug;11(4):235-45.

12. Tyler JL, Yamamoto YL, Diksic M, Theron J, Villemure JG, Worthington C, et al. Pharmacokinetics of superselective intra-arterial and intravenous [11C] BCNU evaluated by PET. J Nucl Med. 1986 Jun; 27(6):775-80.

13. Moehler M, Dimitrakopoulou-Strauss A, Gutzler F, Raeth U, Strauss LG, Stremmel W. 18F-labeled fluorouracil positron emission tomography and the prognoses of colorectal carcinoma patients with metastases to the liver treated with 5-fluorouracil. Cancer. 1998 Jul 15;83(2):245-53.

14. Saleem A, Brown GD, Brady F, Aboagye EO, Osman S, Luthra SK, et al. Metabolic activation of temozolomide measured in vivo using positron emission tomography. Cancer Res. 2003 May 15;63(10): 2409-15.

15. Singh $M$, Waluch $V$. Physics and instrumentation for imaging in-vivo drug distribution. Adv Drug Deliv Rev. 2000 Mar 15;41(1):7-20.

16. Lyoo IK, Renshaw PF. Magnetic resonance spectroscopy: current and future applications in psychiatric research. Biol Psychiatry. 2002 Feb 1;51(3):195-207.

17. Wolf W, Presant CA, Waluch V. 19F-MRS studies of fluorinated drugs in humans. Adv Drug Deliv Rev. 2000 Mar 15;41(1):55-74.

18. Griffiths JR, Glickson JD. Monitoring pharmacokinetics of anticancer drugs: non-invasive investigation using magnetic resonance spectroscopy. Adv Drug Deliv Rev. 2000 Mar 15;41(1):75-89.

19. Caprioli RM, Farmer TB, Gile J. Molecular imaging of biological samples: localization of peptides and proteins using MALDI-TOF MS. Anal Chem. 1997 Dec 1;69(23):4751-60.

20. Schöne $\mathrm{C}$, Höfler H, Walch A. MALDI imaging mass spectrometry in cancer research: combining proteomic profiling and histological evaluation. Clin Biochem. 2013 Apr;46(6):539-45.

21. Stauber J, Lemaire R, Franck J, Bonnel D, Croix D, Day R, et al. MALDI imaging of formalin-fixed paraffin-embedded tissues: application to model animals of Parkinson disease for biomarker hunting. $J$ Proteome Res. 2008 Mar;7(3):969-78.

22. Rohner TC, Staab D, Stoeckli M. MALDI mass spectrometric imaging of biological tissue sections. Mech Ageing Dev. 2005 Jan;126(1):17785.

23. Benabdellah F, Yu H, Brunelle A, Laprévote O, De La Porte S. MALDI 
reveals membrane lipid profile reversion in MDX mice. Neurobiol Dis. 2009 Nov;36(2):252-8.

24. Cazares LH, Troyer D, Mendrinos S, Lance RA, Nyalwidhe J0, Beydoun HA, et al. Imaging mass spectrometry of a specific fragment of mitogen-activated protein kinase/extracellular signal-regulated kinase kinase kinase 2 discriminates cancer from uninvolved prostate tissue. Clin Cancer Res. 2009 Sep 1;15(17):5541-51.

25. Balluff B, Elsner M, Kowarsch A, Rauser S, Meding S, Schuhmacher $C$, et al. Classification of HER2/neu status in gastric cancer using a breast-cancer derived proteome classifier. J Proteome Res. 2010 Dec 3:9(12):6317-22.

26. Balluff B, Rauser S, Meding S, Elsner M, Schone C, Feuchtinger A, et al. MALDI imaging identifies prognostic seven-protein signature of novel tissue markers in intestinal-type gastric cancer. Am J Pathol. 2011 Dec;179(6):2720-9.

27. Djidja MC, Francese S, Loadman PM, Sutton CW, Scriven P, Claude E, et al. Detergent addition to tryptic digests and ion mobility separation prior to MS/MS improves peptide yield and protein identification for in situ proteomic investigation of frozen and formalin-fixed paraffinembedded adenocarcinoma tissue sections. Proteomics. 2009 May; 9(10):2750-63.

28. El Ayed M, Bonnel D, Longuespée R, Castelier C, Franck J, Vergara D, et al. MALDI imaging mass spectrometry in ovarian cancer for tracking, identifying, and validating biomarkers. Med Sci Monit. 2010 Aug;16(8):BR233-45.

29. Castellino S, Groseclose MR, Wagner D. MALDI imaging mass spectrometry: bridging biology and chemistry in drug development. Bioanalysis. 2011 Nov;3(21):2427-41.

30. Morosi L, Zucchetti M, D'Incalci M, Davoli E. Imaging mass spectrometry: challenges in visualization of drug distribution in solid tumors. Curr Opin Pharmacol. 2013 0ct;13(5):807-12.

31. Reyzer ML, Hsieh Y, Ng K, Korfmacher WA, Caprioli RM. Direct analysis of drug candidates in tissue by matrix-assisted laser desorption/ionization mass spectrometry. J Mass Spectrom. 2003 Oct;38(10):1081-92.

32. Hsieh Y, Chen J, Korfmacher WA. Mapping pharmaceuticals in tissues using MALDI imaging mass spectrometry. J Pharmacol Toxicol Methods. 2007 Mar-Apr;55(2):193-200.

33. Cornett DS, Frappier SL, Caprioli RM. MALDI-FTICR imaging mass spectrometry of drugs and metabolites in tissue. Anal Chem. $2008 \mathrm{Jul}$ 15;80(14):5648-53.

34. Atkinson SJ, Loadman PM, Sutton C, Patterson LH, Clench MR. Examination of the distribution of the bioreductive drug AQ4N and its active metabolite AQ4 in solid tumours by imaging matrix-assisted laser desorption/ionisation mass spectrometry. Rapid Commun Mass Spectrom. 2007;21(7):1271-6.

35. Huamani J, Willey C, Thotala D, Niermann KJ, Reyzer M, Leavitt L, et al. Differential efficacy of combined therapy with radiation and AEE788 in high and low EGFR-expressing androgen-independent prostate tumor models. Int J Radiat Oncol Biol Phys. 2008 May 1;71(1):23746.

36. Marko-Varga G, Fehniger TE, Rezeli M, Döme B, Laurell T, Végvári A. Drug localization in different lung cancer phenotypes by MALDI mass spectrometry imaging. J Proteomics. 2011 Jun 10;74(7):982-92.

37. Fehniger TE, Végvári A, Rezeli M, Prikk K, Ross P, Dahlbäck M, et al. Direct demonstration of tissue uptake of an inhaled drug: proof-ofprinciple study using matrix-assisted laser desorption ionization mass spectrometry imaging. Anal Chem. 2011 Nov 1;83(21):8329-36.

38. Marko-Varga G. BioBanking as the central tool for translational medicine CTM issue 2013. Clin Transl Med. 2013 Feb 6;2(1):4.

39. Yasunaga M, Furuta M, Ogata K, Koga Y, Yamamoto Y, Takigahira M et al. The significance of microscopic mass spectrometry with high resolution in the visualisation of drug distribution. Sci Rep. 2013 Oct 25;3:3050.

40. Morosi L, Spinelli P, Zucchetti M, Pretto F, Carra A, D'Incalci M, et al. Determination of paclitaxel distribution in solid tumors by nanoparticle assisted laser desorption ionization mass spectrometry imaging. PLoS One. 2013 Aug 26;8(8):e72532.

41. Solon EG, Schweitzer A, Stoeckli M, Prideaux B. Autoradiography, MALDI-MS, and SIMS-MS imaging in pharmaceutical discovery and development. AAPS J. 2010 Mar;12(1):11-26.

42. Minchinton Al, Tannock IF. Drug penetration in solid tumours. Nat Rev Cancer. 2006 Aug;6(8):583-92.

43. Trédan 0, Galmarini CM, Patel K, Tannock IF. Drug resistance and the solid tumor microenvironment. J Natl Cancer Inst. 2007 Oct 3;99(19): 1441-54. Epub 2007 Sep 25.

44. Lankelma J, Dekker H, Luque FR, Luykx S, Hoekman K, van der Valk $P$, et al. Doxorubicin gradients in human breast cancer. Clin Cancer Res. 1999 Jul;5(7):1703-7.

45. Stumpf WE. Whole-body and microscopic autoradiography to determine tissue distribution of biopharmaceuticals -- target discoveries with receptor micro-autoradiography engendered new concepts and therapies for vitamin D. Adv Drug Deliv Rev. 2013 Jul;65(8):1086-97.

46. Kyle AH, Huxham LA, Yeoman DM, Minchinton Al. Limited tissue penetration of taxanes: a mechanism for resistance in solid tumors. Clin Cancer Res. 2007 May 1;13(9):2804-10. 